\title{
Recurrent respiratory tract infections in children; beyond medical causes!
}

Keywords: recurrent respiratory tract infections, tracheaesophageal fistula, congenital lobar emphysema, surgical causes, foreign body trachea

\section{Letter to the editor}

In today's world an increasing number children are presenting with respiratory complaints like respiratory distress, dyspnoea, cough, and recurrent respiratory tract infections (RTI), with their cause being traced to conditions beyond common pediatric medical disease (Table 1). A few such cases and their related causes are highlighted below. An eighteen months old boy presented to the emergency with severe pneumonia and consolidation of the right upper lobe. He was admitted by the paediatrician, started intravenous antibiotics and required ventilator support. A paediatric surgery referral was sent in view of history of repeated admissions for severe recurrent RTI every 3-4 weeks since birth. A flexible endoscopic examination revealed a fistulous opening in the posterior wall of the trachea raising the suspicion of an $\mathrm{H}$ type trachea-oesophageal (TEF) fistula. On deeper probing, the mother gave a history of choking during feeds. After proper stabilization rigid bronchoscope under general anaesthesia revealed a fistula in the posterior wall of trachea which was first cannulated, and then ligated and repaired from the right cervical route (Figure 1). The child was discharged after 10 days and was followedup for the next 10 months. He had no other episode of RTI which required admission

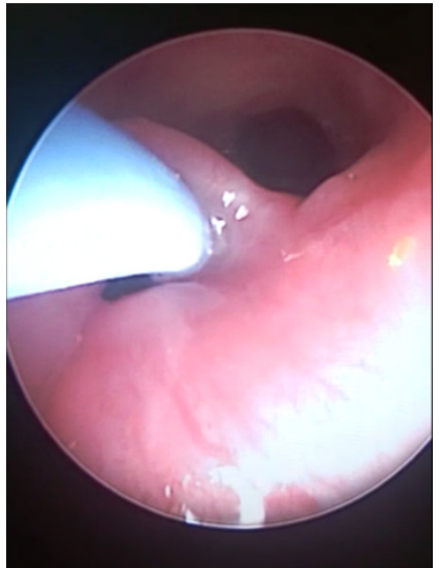

Figure I Rigid bronchoscopy showed an opening in the posterior wall of the trachea which was cannulated using ureteric catheter.

Another 4 months old child admitted with history of recurrent RTI since birth with completely asymptomatic intervening periods. In the previous episode, the child was admitted with severe respiratory distress and was treated by left sided inter-costal tube drainage in view of left pneumothorax causing meditational shift (Figure $2 \mathrm{~A}$ ). A CECT scan of the chest revealed hyper-lucent left sided lung field with mediastinal shift towards the right side (Figure 2B). The left lower lobe remained collapsed with depressed left hemi-diaphragm. This child underwent left postero-lateral thoracotomy and left upper

\author{
Volume 8 Issue 4 - 2018
} Kashish Khanna, Shilpa Sharma, Devendra K
Gupta

Department of Pediatric Surgery, All India Institute of Medical Sciences, India

Correspondence: Devendra K Gupta, Professor and Head, Department of Pediatric Surgery, All India Institute of Medical Sciences, New Delhi-I I 0029, India, Tel 0I I-26593309, FAX 0 I I26588663,Email profdkgupta@gmail.com

Received: December 20, 2017| Published: August 13, 2018

lobotomy (Figure 3A). The child had congenital lobar emphysema of the left upper lobe (Figure 3B).

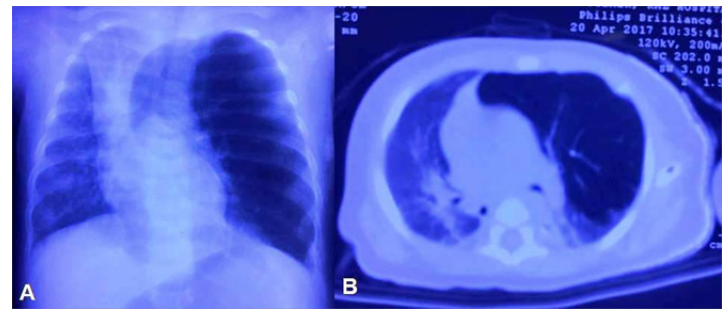

Figure 2A-Chest $\mathrm{X}$-ray with hyper lucent left lung field extending across the mediastinal into the right side with retained broncho vascular markings suggestive of congenital lobar emphysema. 2B- CT scan suggestive of cystic lung lesion with collapse of the left lower lobe.

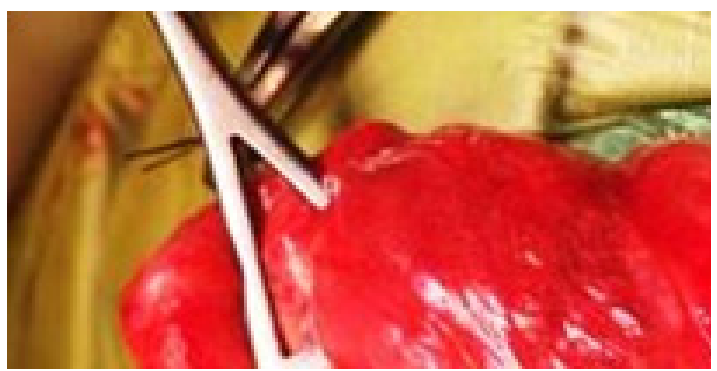

Figure 3A- Left postero-lateral thoracotomy and left upper lobectomy done in a 4 month old boy for cystic lung lesion. 3B-Gross specimen and histology suggestive of congenital lobar emphysema of the left upper lobe.

A 3-month-old girl presented with complaints of respiratory distress since 1.5 months and 1 episode of bloody expectoration 3 days back. On examination, she had tachycardia and tachypnea and chest auscultation revealed decreased air entry in the left upper zone. ${ }^{1}$ The $\mathrm{X}$-Ray chest was suggestive of a cavity lesion in the left upper lobe with surrounding area of consolidation. A Tc-99m pertechnetate scan showed avid uptake in this lesion suggestive of foregut duplication cyst. ${ }^{1}$ This child underwent left posterolateral thoracotomy and excision of the thoracic part of a ruptured neuroenteric cyst. Many a time paediatricians encounter children who suffer from repeated life- 
threatening RTI which may present acutely as respiratory distress or chronically as repeated chest infections. While a detailed work-up rule out medical causes, some surgical causes masquerading as recurrent RTI as cited above require special mention. Table 1 summarizes most of these causes which could be treated surgically. These can broadly be classified according to their organ of origin. ${ }^{2}$

A list of surgical causes masquerading as recurrent respiratory tract infections in pediatric age group

\begin{tabular}{|c|c|c|}
\hline S No & $\begin{array}{l}\text { Causes } \\
\text { related to }\end{array}$ & Anomalies or diseases \\
\hline \multirow[t]{5}{*}{ I } & $\begin{array}{l}\text { Tracheo- } \\
\text { bronchial tree }\end{array}$ & Tracheomalacia \\
\hline & & Tracheal stenosis \\
\hline & & Tracheal bronchus \\
\hline & & Bronchial atresia \\
\hline & & Bronchogenic cyst \\
\hline \multirow[t]{7}{*}{2} & Lung & Pulmonary agenesis \\
\hline & & Pulmonary aplasia \\
\hline & & Pulmonary hypoplasia \\
\hline & & Scimitar syndrome \\
\hline & & $\begin{array}{l}\text { Congenital cystic adenomatoid malformation } \\
\text { (CCAM) }\end{array}$ \\
\hline & & Congenital lobar emphysema (CLE) \\
\hline & & Pulmonary sequestration \\
\hline \multirow[t]{2}{*}{3} & $\begin{array}{l}\text { Esophageal } \\
\text { anomalies }\end{array}$ & $\begin{array}{l}\text { Esophageal atresia and trachea-oesophageal } \\
\text { fistula ( } \mathrm{H} \text { type/ } \mathrm{N} \text { type) }\end{array}$ \\
\hline & & Esophageal duplications \\
\hline \multirow[t]{2}{*}{4} & Diaphragmatic & Congenital diaphragmatic hernia \\
\hline & & Eventration of diaphragm \\
\hline \multirow[t]{4}{*}{5} & Mediastinum & Thymic lesions \\
\hline & & Thyroid masses \\
\hline & & Thymolymphatic cysts \\
\hline & & $\begin{array}{l}\text { Tumors (germ cell tumours, neuroblastomas, } \\
\text { lymphoma) }\end{array}$ \\
\hline 4 & $\begin{array}{l}\text { Vascular } \\
\text { anomalies }\end{array}$ & Vascular rings \\
\hline \multirow[t]{5}{*}{5} & Miscellaneous & Foreign body \\
\hline & & Dermoid, epidermoid \\
\hline & & Lipoma \\
\hline & & Post Corrosive injury \\
\hline & & Post traumatic \\
\hline
\end{tabular}

\section{Airway related problems}

A. Foreign body (FB) trachea: FBs in children range from commonly aspirated peanuts, other nuts, seeds, food particles, parts of whistle, hardware, parts of toys and uncommon FBs like paint particles, safety pins, rubber balloons and marbles. ${ }^{3}$ Seeds like that of tamarind are the most dangerous types of FBs as they swell-up with time and covert partial obstruction to complete airway obstruction. Other factors like sharp objects, rounded objects, corrosive batteries and brittle objects are again problematic. Child may present acutely with airway obstruction or with a history of choking or cyanotic episode a few days ago or with RTI due to long standing impacted trachea-bronchial FB. After confirming the diagnosis by a plain radiograph and occasionally a $\mathrm{CT}$ chest, a rigid bronchoscope by an experienced surgeon with availability of post-op Ventilatory care can take care of most aspirated foreign bodies.

B. Tracheomalacia: Tracheomalacia is a condition where the weakening in the wall of the trachea causes it to collapse partially during expiration. This may be congenital or secondary to a surgery in the neck. Children often present with symptoms mimicking asthma namely; wheeze, cough, stridor, dyspnoea, cyanosis and recurrent RTI. Cine CT scan of the airway or a bronchoscopy reveals the weakness in the tracheal wall. Aortopexy remains the treatment of choice in severe cases. ${ }^{4}$

C. Tracheal stenosis

D. Tracheal diverticula

\section{Esophageal anomalies}

a) Esophageal atresia with tracheoesophageal fistula (EA-TEF): Most TEF are usually detected at birth. $\mathrm{H}$ type EA-TEF constitutes $4 \%$ of the TEF anomalies and are classified as Type E TEF. ${ }^{2}$ The fistula follows an oblique course from the posterior wall of the trachea cranially and anteriorly to the anterior wall of the esophagus caudally and posteriorly. Delayed diagnosis is usual due to lack of suspicion, rarity of this condition and overlap of symptoms with other medical illness. A cine-esophagogram is the usual investigation of choice, though a brochoesophagoscopy is diagnostic. A non-ionic water soluble contrast or properly diluted barium can be used for the contrast study. The child is positioned in a prone position and an infant feeding tube is withdrawn from the lowermost point in the tracheobronchial tree at a constant rate. Meanwhile, contrast is injected under pressure continuously and multiple rapid sequence films or a cine-esophagogram is obtained to diagnose an $\mathrm{H}$ type TEF. ${ }^{2}$ The bronchoscopy provides additional advantage of localizing the fistula and passing a Fogarty catheter or a ureteric catheter which would later aid during surgery. Surgery consists of fistula ligation, division and repair of defects on tracheal as well as oesophageal side.

b) Esophageal Duplication: Esophageal duplication cysts whether tubular or cystic may cause compression of the adjacent lung parenchyma or the native esophagus and thus present with respiratory difficulty, dysphagia or vomiting. ${ }^{3}$ A CT chest with well marginated, homogenously intense, non enhanced spherical swelling, usually in the right hemi-thorax is suggestive of duplication cysts. A Tc-99m-pertechnetate scan showing uptake due to the presence of ectopic gastric mucosa is diagnostic of duplication cysts. These cysts when associated with vertebral fusion defects are most commonly associated with neuroenteric cysts. ${ }^{1}$

\section{Lung related diseases}

a) Congenital lobar emphysema (CLE): CLE is the isolated 
idiopathic hyperinflation of a lobe of the lung. The left upper lobe is most commonly involved. At birth, this segment is filled with fluid and appears opaque on a radiograph. With time, the fluid gets absorbed and the air gets trapped, the affected lobe becomes hyper inflated and appears translucent on radiograph films. There may be compression of adjacent lobes and shifting of the mediastinum in worst cases. It can be differentiated from pneumothorax by the presence of broncho vascular markings in CLE. The fear of repeated chest infections and respiratory distress calls for lobectomy of the affected segment of the lung.

b) Congenital Pulmonary airway malformations (CPAM): These are solid/cystic lung lesions which can be diagnosed on prenatal ultrasound. If missed, they present in the first few years of life with repeated chest infections. Chest $\mathrm{X}$ ray shows multiple airfilled thinned walled cysts of various sizes. ${ }^{5} \mathrm{CT}$ chest better characterizes the nature and location of CPAM. Lobectomy of the concerned segment is the surgery of choice.

c) Pulmonary sequestration: It is a segment of lung without anatomic bronchial communication to the lung. It receives its blood supply from the systemic circulation either from the thoracic or the abdominal aorta. The Extralobar sequestrationis often detected incidentally and is associated with $\mathrm{CDH}$. The Intralobar sequestration is found within the normal lung parenchyma most commonly in the lower lobes. Lung sequestrations are prone to infection so they generally do not present as acute emergency at birth. When encountered, CT angiography to establish their systemic arterial supply should be done before planning resection.

d) Bronchogenic cyst: Congenital cystic foregut malformations found in the hilum, mediastinum, or within the lung parenchyma. These can compress the airway and cause atelectasis, pneumonia and air trapping. They do not usually communicate with the trachea-bronchial tree and present later in infancy and childhood.

\section{Diaphragmatic hernias}

a) Congenital Diaphragmatic hernia (CDH): A newborn with $\mathrm{CDH}$ may be diagnosed antenatally or may present with respiratory distress with or without cyanosis just after birth or at a later age. Clinically, the child will have a scaphoid abdomen, shifting of the heart sounds and gurgling bowel sounds can be heard in the chest. An X-ray with bowel loops seen in the chest clinches the diagnosis of CHD. Bag and mask ventilation should not be done. Immediate nasogastric tube decompression, rectal syringing and saturation monitoring should be started. $\mathrm{CDH}$ is not a surgical emergency. Surgery can be planned once adequate ventilator control has been reached. Most CDH are left sided
$(80 \%)$ posterolateral defects or the Bochdalek's hernia and are repaired primarily via the thoracotomy approach. Occasionally patches (artificial or tissue patch) are required in cases of large defects. Morgagni or the anterior defect often present late in life and can easily be repaired thoracoscopically. Persistent fetal circulation, hypoplastic lungs and pulmonary hypertension are the greatest challenges in the management of $\mathrm{CDH}$.

b) Eventuation of the diaphragm: Usually present within the first few years of life. There is herniation of the bowel loops into the thoracic cavity in the absence of an actual defect but rather due the muscular weakness of the diaphragm. It can also occur after injury to the phrenic nerve. Diaphragmatic repair by open or thoracoscopic approach is required.

\section{Vascular anomalies}

Vascular rings whether complete or incomplete may cause compressive symptoms like dysphagia or respiratory distress. An X-ray chest or an esophagogram may give important clues. However a CT angiogram or an MR imaging confirms the diagnosis. A double aortic arch is seen as bilateral tracheal indentation on a plan X-ray chest. ${ }^{2}$ Most of these congenital lesions are associated with other VACTERL (Vertebral, Anorectal, Cardiac, Tracheo-oesophageal, Renal and Limb anomalies). Hence once the acute respiratory condition has been dealt with, a physician must rule out any other associated anomalies.

\section{Acknowledgements}

None.

\section{Conflict of interest}

The author declares there is no conflict of interest.

\section{References}

1. Gundapaneni S, Jain V, Sharma S, et al. Perforated neuroenteric cyst masquerading as congenital pulmonary airway malformation. BMJ Case Rep. 2017.

2. Berrocal T, Madrid C, Novo S, et al. Congenital anomalies of the tracheobronchial tree, lung and mediastinum: embryology, radiology, and pathology. Radio graphics. 2004;24(1):e17.

3. Altuntas B, Aydın Y, Eroglu A. Foreign Bodies in Trachea: A 25-years of Experience. The Eurasian J Med. 2016;48(2):119-123.

4. Weber TR, Keller MS, Fiore A. Aortic suspension (aortopexy) for severe tracheomalacia in infants and children. Am J Surg.2002;184(6):573-577.

5. JT Stocker. Cystic lung disease in infants and children. Fetal Pediatr Pathol. 2009;28(4):155-184. 